\title{
PROOF OF GOLDBACH CONJECTURE
}

\author{
Ren Yong Xue, Ren Yi \\ China's Inner Mongolia reemployment of dental group g stone sing-an oil company Inner Mongolia \\ teeth, 022150 \\ and \\ College of preventive medicine specialty in south China's guangdong province, \\ Guangzhou, 510310
}

Keywords: create double coordinate system; the sum of a prime number; Infinite sets; generalized integral value; Equations; inference

\begin{abstract}
This paper through the creation of "double rectangular coordinate system", yin quad ints, each coordinate axis coordinates all constitute the infinite sets. Coordina es with infin s one toone correspondence between the elements within and equal relations Witt any $f$, sum of two odd prime Numbers $(a+b)$ to form a square area, length for ya of a so are area A1 "square" diagonal integration method, a quarter of a square area 1 equatio d rived with the definite integral equation; A1 area value of the argument as in nit neralized stegral value, and deduce the equations, the Goldbach conjecture.
\end{abstract}

\section{INTRODUCTION}

The content of the Goldbach conjecture:

(a) Any even number greater than or equal 6 can be us the sum of two odd prime Numbers.

(b) Any odd integer greater than or equal 0 primeNumbers.

This is the Goldbach conjecture

Proof: Goldbach conjecture

(a) $2 \mathrm{n}=\mathrm{a}+\mathrm{b}$

(b) $2 \mathrm{n}-1=\mathrm{a}+\mathrm{b}+\mathrm{c}$

Equation $2 \mathrm{n}=\mathrm{a}+\mathrm{b}$, Must sa $\mathrm{v}$ the following conditions:

Firstly, the $\mathrm{n}$ is gre ater than or equ, $/ 3$ any element of the set of natural Numbers is infinite.

Set: $\mathrm{n} 3$ natural $\mathrm{Nu}$ ers or in mite collection of symbols for $2 \mathrm{n} \in 2(\mathrm{~N}+\geqq 3), \mathrm{n} \in \mathrm{n}+\geqq 3$.

Second: $b$ is o rim os infinite any two elements in the collection. $a$ and $b$ in the odd primes within he int ite set ar irary values.

Set: odd p es infinite collection of symbols for "Jss", $a \in$ Jss, $\in$ b Jss

Equations: $2 \quad a+b$ satisfies conditions: $a \in$ Jss, $\in$ b Jss, a 3 or higher or $3 \mathrm{~b}$,

$\mathrm{N} \in \mathrm{n}+\geqq 3, \mathrm{n} \in\{3,4,5 \ldots\}, 2 \in \mathrm{N}(\mathrm{N}+2 \geqq 3)$, conform to any an even number greater than or equal to 6 can be expressed as the sum of two odd prime Numbers.

Equation (b) $2 \mathrm{n}-1=\mathrm{a}+\mathrm{b}+\mathrm{c}$ conditions must be satisfied:

Firstly, the $\mathrm{n}$ is greater than or equal to 5 infinite any one element of a set of natural Numbers.

Set: $\mathrm{n}$ p 5 natural Numbers is infinite collection of symbols for " $\mathrm{n}+\geqq 5$ ";

$\mathrm{N} \in \mathrm{n}+\geqq 5$

Second: a, b, c are odd prime Numbers is infinite any three elements in the collection, a, b, c in odd primes infinite set arbitrary values.

Set odd primes infinite sets symbol for "Jss" 
$\mathrm{A} \in \mathrm{Jss}, \in \mathrm{b}$ Jss, $\mathrm{c} \in \mathrm{Jss}$

Equations, (b) $(2 \mathrm{n}-1=\mathrm{a}+\mathrm{b}+\mathrm{c}$ conditions must be satisfied:

A 3 or higher, $\mathrm{b} 3$ or higher, $\mathrm{c} 3$ or higher; $\mathrm{A} \in \mathrm{Jss}, \in \mathrm{b}$ Jss

$\mathrm{C} \in \mathrm{Jss}, \mathrm{n} \in \mathrm{n}+\geqq 5, \mathrm{n} \in\{5,6,7,8 \ldots\}$, conform to any odd integer greater than or equal to 9 , can be expressed as the sum of three odd prime Numbers.

2. Prove Goldbach conjecture: create a new "double rectangular coordinate system is shown infigure 1 .

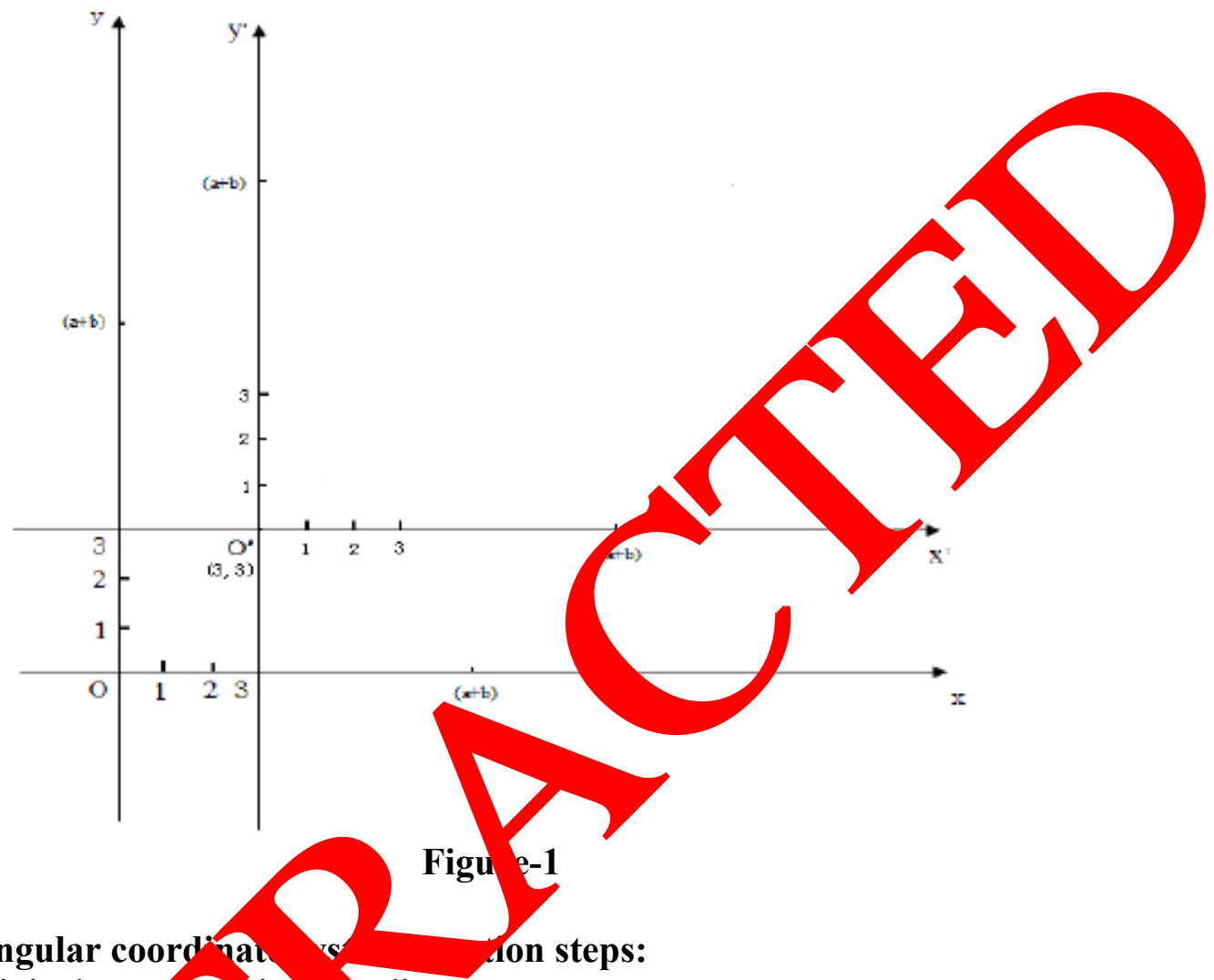

2.1 Double rectangular coord 7 a

1 , based on the original xoy artesian rdinate system

2 , OS $x$ axial oy axis vert ca prizontal in the position of the odd prime number 3

3 , oy axial OS $x$ axis a ection orpendieular to the horizontal displacement to the position of the odd prime number

4, OS $\mathrm{x}$ axis an oy as perpend,cular intersection in the origin of the new position of the odd prime

number 3 , in the $\mathrm{o}$ nal co ordinates xoy within the first quadrant, established a new coordinate system, 0 ,

Xoy he a ginal co ordinate system and the new coordinate system x, 'o, y', both together constituı vum dangular coordinate system", as shown in figure 1.

2.2 in doubly quadrant in the rectangular coordinate system, the axes of the coordinate values and the relationship between the corresponding collections, meet such as under the given conditions:

1 , on the OS $\mathrm{x}$ axis and oy shaft:

There is one-to-one correspondence and equal by all the natural Numbers for infinite sets of elements. As "N". There is a one-to-one correspondence and equal infinite set by all the evennumber of elements. As " $2 \mathrm{n}$ left". There is a one-to-one correspondence and equal by all the prime Numbers is infinite sets of elements. As "Ss".

2, in $\mathrm{o}, \mathrm{x}$ and $\mathrm{o}, \mathrm{y}^{\prime}$ shaft: There is a one-to-one correspondence and equal by natural number (positive integer) is greater than or equal to 3 for infinite sets of elements. As $\mathrm{N}+3$ or more or $(\mathrm{Z}+$ 
3 or higher) There is one-to-one correspondence and equal by all the odd prime number for the infinite sets of elements. Remember to make "the Jss" There is a one-to-one correspondence and equal by any of the sum of two odd prime Numbers $(a+b)$ for the infinite sets of elements. As " 2 $(\mathrm{N}+3$ or higher)" There is one-to-one correspondence and equal by three odd prime number $(\mathrm{a}+\mathrm{b}$ $+c)$ is greater than or equal to the sum of nine odd for infinite sets of elements. As "Js 9 or higher. There is a one-to-one correspondence and equal by the sum of any three odd prime, plus $1,(\mathrm{a}+\mathrm{b}+$ $\mathrm{c}+1$ ) for the infinite sets of elements. As "2

\subsection{Within the I quadrant, the coordinate values with an infinite set of relationships between elements known to satisfy the following conditions:}

1 , the coordinate values on each axis, with its corresponding one to one corresponden hetween infinite sets of elements and equal relationship. Axes coordinate values of the gometry the performance of a zero dimensional "points".

2, any of the sum of two odd prime Numbers $(a+b)$ on each axis corresponding infinite collection there is one-to-one correspondence be reen t eleme within and equal relationship. $(\mathrm{a}+\mathrm{b})$ on the axis of coordinates of the al fig show the onedimensional"line".

3 , in the $\mathrm{x}$ ' $\mathrm{o}, \mathrm{y}$ ', $(\mathrm{a}+\mathrm{b})$ to a side length of square area, with $\mathrm{y}(\mathrm{a}$ is a side length of square area, there is a one-to-one correspondence and equal rel uromip, with an form an infinite set ofan element. $(a+b)$ for the length of the squar of the geometre figure is shown as a twodimensional "face".

2.4 Within I quadrant, with any of the sum of two length $(a+b)$, consisting of a square area $A$, in of equation is derived.

$\because a \in$ Jss ; $b \in$ Jss $a \geq 3 b \geq 3$; known: $x=(a+b) \quad y=$ (a); Function: $y=x ; A=2 S 1 ;(a+b) \geq 6$; $(\mathrm{a}+\mathrm{b}) \in 2\left(\mathrm{~N}^{+} \geq 3\right)$

As A special curved trapezoid area $=(a+b)$ and $x$ axis and $y=$ cos a triangic $S 1$ in $y$ axis on $[0,(a+b)]$, composed of $x=(a+$ $b$ ), and $y=x$ and $y$ axis $r$ triangle side length for $(a+b)$ a. square area (square area $a$ is equal to 2 times the rig, trian area of $S_{1}$ ).

$\mathrm{A}=\mathrm{S}_{1}+\mathrm{S}_{2}$

According to on, 1 -

If the function $\mathrm{f}(\mathrm{x}$, continy $\mathrm{us}$ on $[\mathrm{a}, \mathrm{b}]$, and there is the function $\mathrm{f}(\mathrm{x})$, then $\mathrm{f}(\mathrm{x})$ integrable on $[\mathrm{a}$, b], and $B$ r limi ower limit) $f(x) d x=f(b)-(a) f$

Laund funct on $y=x,[0,(a+b)]$ on continuously, and the function $F(x)=x 2 / 2$, the function $y$

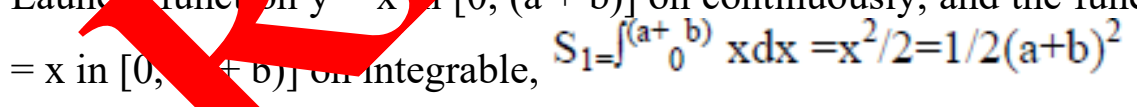

$\therefore$ with definite integral is expressed as $\mathrm{A}: \mathrm{A}=2 \mathrm{~S}_{1} ; \mathrm{A}=2 \int_{0}^{(\mathrm{a}+\mathrm{b})} \mathrm{xdx}=(\mathrm{a}+\mathrm{b})^{2}$

Proof: in $[0,+\infty)$, A generalized integral for infinite range of values.

Proof:

Known:

$1, \mathrm{y}=\mathrm{x}$ is the elementary function, define the interval $[0,+\infty)$ for continuous function, meet the function continuous condition.

$2, a \in$ Jss,$b \in$ Jss $, a \geq 3, b \geq 3 ; y=x$ function $F(x)=1 / 2(x)^{2}$

$(a+b) \geq 6,(a+b) \in 2\left(N^{+} \geq 3\right) ;$ By $y=(a+b), x=(a+b)$ and $y=x$ of a right triangle S1 of the generalized integral, 
$\mathrm{F}(\mathrm{x})=1 / 2 \mathrm{x} 2$ is an antiderivative of $\mathrm{y}=\mathrm{x}$,

$\int_{0}^{+\infty} \mathrm{xdx}=\lim \int_{0}^{(\mathrm{a}+\mathrm{b})} \mathrm{xdx}=\lim \left(\mathrm{x}^{2} / 2\right)=\lim 1 / 2(\mathrm{a}+\mathrm{b})^{2}$

$\mathrm{S}_{1}=$

$$
(\mathrm{a}+\mathrm{b}) \rightarrow+\infty \quad \mathrm{x} \rightarrow+\infty \quad(\mathrm{a}+\mathrm{b}) \rightarrow+\infty
$$

$=1 / 2(\mathrm{a}+\mathrm{b})^{2}$

$\mathrm{A}=2 \mathrm{~S}_{1}$;

$$
\begin{aligned}
& 2 \int_{0}^{+\infty} \mathrm{xdx}=\left.\lim \right|_{0} ^{+\infty} 2 \mathrm{xdx}=\lim 2\left[\mathrm{x}^{2} / 2\right]=\lim 2\left[(\mathrm{a}+\mathrm{b})^{2} / 2\right]=(\mathrm{a}+\mathrm{b})^{2} \\
& \mathrm{~A}=\quad(\mathrm{a}+\mathrm{b}) \rightarrow+\infty \quad \mathrm{x} \rightarrow+\infty \quad(\mathrm{a}+\mathrm{b}) \rightarrow+\infty
\end{aligned}
$$

\section{Conclusion 1:}

In any of the sum of two odd prime Numbers $(a+b)$ to the area of the side length of sa is of value as the limit of infinite generalized integral, as shown in figure 2

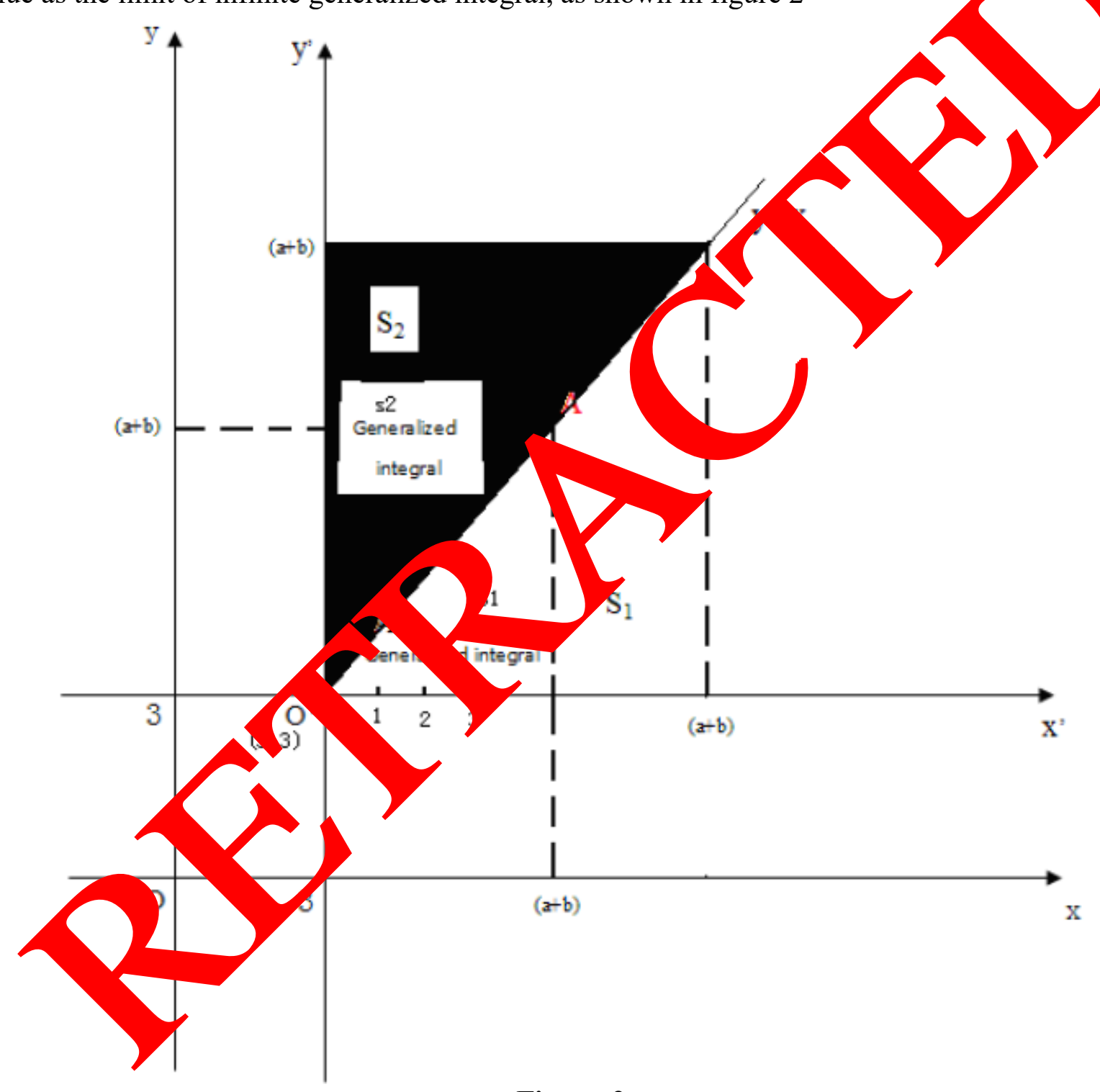

Figure-2

2.5 Within I quadrant to $(a+b) / 2$ is a side length of the midpoint coordinates, the $a$ is divided into four small

Square area (see figure 3), 


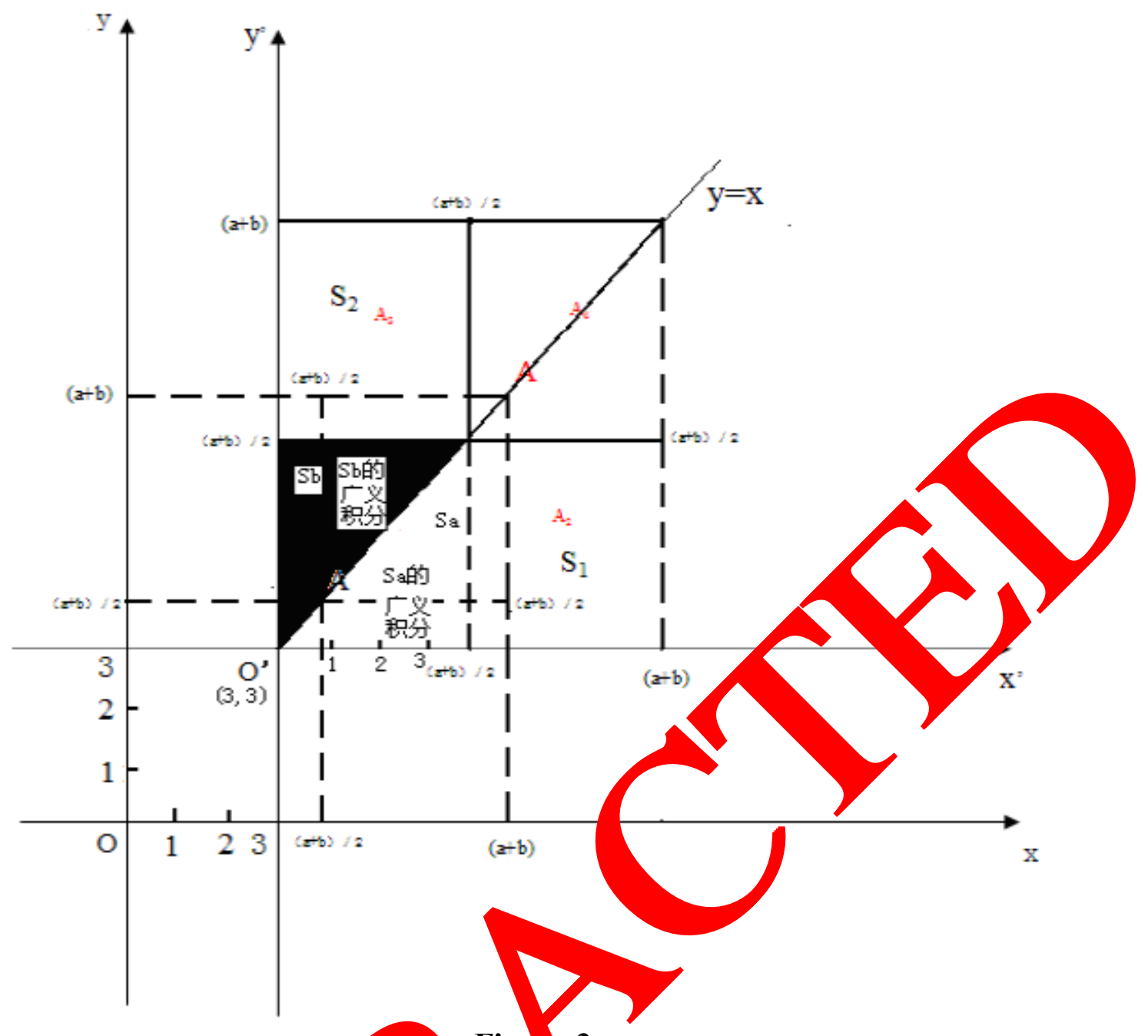

Make the $A_{1}=A_{2}=A_{3}=A_{4}=$ quture area $A 1$ equations is derived. $\mathrm{y}=(\mathrm{a}+\mathrm{b}) / 2$;

$$
\mathrm{X}=(\mathrm{a}+\mathrm{b}) / 2 ; \mathrm{y}=\mathrm{x}, \mathrm{A}_{1}=2, \mathrm{~S}_{\mathrm{a}}=\mathrm{S}_{\mathrm{b}}
$$

$\mathrm{a} \in \mathrm{Jss}, \mathrm{b} \in \mathrm{Jss} \quad \geq 3, \mathrm{~b}-3,\left(\mathrm{a}+\mathrm{b}, \geq 6,(\mathrm{a}+\mathrm{b}) / 2 \geq 3,(\mathrm{a}+\mathrm{b}) / 2 \in \mathrm{N}^{+} \geq 3\right.$

See $A_{1}$ as a spec coved ti pezoid area, $y=x$ in the interval $[0,(a+b) / 2]$ on integrable, $A_{1}$ expressed in-definit tegral $s$ :

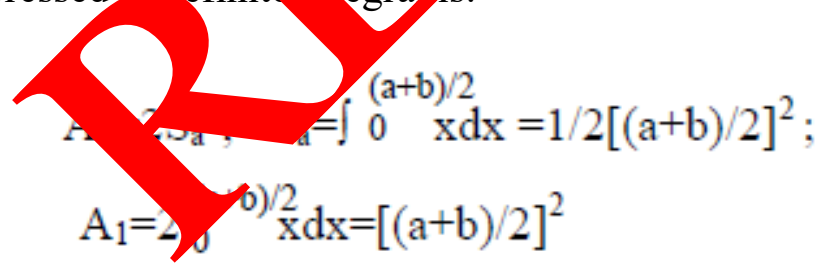

Proof: in $[0,+\infty), \mathrm{A} 1$ as the limit of infinite generalized integral.

Proof:

Known:

$1, \mathrm{y}=\mathrm{x}$ is the elementary function, define the interval $[0,+\infty)$ for continuous function, meet the function continuous condition.

2. $a \in$ Jss,$b \in J$ Js $, a \geq 3, b \geq 3 ; y=(a+b) / 2$;

$$
\begin{aligned}
& \mathrm{X}=(\mathrm{a}+\mathrm{b}) / 2 ; \mathrm{y}=\mathrm{x} ; \mathrm{A} 1=2 \mathrm{~S}_{\mathrm{a}} ; \mathrm{S}_{\mathrm{a}}=\mathrm{S}_{\mathrm{b}} ; \\
& \quad(\mathrm{a}+\mathrm{b}) \geq 6,(\mathrm{a}+\mathrm{b}) / 2 \geq 3,(\mathrm{a}+\mathrm{b}) / 2 \in \mathrm{N}^{+} \geq 3
\end{aligned}
$$


$\mathrm{Y}=\mathrm{x}$ function $\mathrm{F}(\mathrm{x})=1 / 2(\mathrm{a}+\mathrm{b})][2 / 2 ; 2$ times the value of the function $21 / 2(\mathrm{a}+\mathrm{b})][2 / 2$ is 9 ormore constant

$(\operatorname{limC}=C) ;$ By $y=(a+b) / 2, x=(a+b) / 2$, and $y=x$ of a right triangle Sa generalized integral; Function $\mathrm{y}=\mathrm{x}$ in $[0,+\infty)$ on the generalized integral. Remember to:

$$
\begin{aligned}
& \quad \int_{0}^{+\infty} \mathrm{xdx}=\lim _{(\mathrm{im}} \int_{0}^{(\mathrm{a}+\mathrm{b}) / 2} \mathrm{xdx}=\lim \left(\mathrm{X}^{2} / 2\right)=\lim 1 / 2[(\mathrm{a}+\mathrm{b}) / 2]^{2} \\
& \mathrm{Sa}=\quad \mathrm{a}+\mathrm{b}) / 2 \rightarrow+\infty \quad \mathrm{x} \rightarrow+\infty \quad(\mathrm{a}+\mathrm{b}) \rightarrow+\infty \\
& =1 / 2[(\mathrm{a}+\mathrm{b}) / 2]^{2} \\
& \mathrm{~A}_{1}=2 \mathrm{~S}_{\mathrm{a}} \\
& \quad 2 \int_{0}^{+\infty} \mathrm{xdx}=\lim \int_{0}^{(\mathrm{a}+\mathrm{b}) / 2} 2 \mathrm{xdx}=\lim \left[2\left(\mathrm{X}^{2} / 2\right)\right]=\lim 2[(\mathrm{a}+\mathrm{b}) / 2]^{2} / 2 \\
& \mathrm{~A}_{1}=\quad(\mathrm{a}+\mathrm{b}) / 2 \rightarrow+\infty \quad \mathrm{x} \rightarrow+\infty \\
& =[(\mathrm{a}+\mathrm{b}) / 2] 2 \\
& \text { Launch of equations: } \left.2 \sqrt{ } \mathrm{A}_{1}=\mathrm{a}+\mathrm{b}\right)
\end{aligned}
$$

Corollary 1:

Any of the sum of two odd prime Numbers $(a+b)$ is equal to t Numbers $(a+b)$ for a quarter of a square a square of side length 2 nes.

Corollary 2:

Any the sum of two odd prime Numbers $(a+b)$ is Numbers $(a+b)$ for a quarter of a square of side leng square root of 2 times.

Launch:

$2 \sqrt{ } \mathrm{A} 1=\mathrm{a}+\mathrm{b}$ Meet the known conditions:

$\sqrt{ } \mathrm{A} 1=(\mathrm{a}+\mathrm{b}) / 2$;

$\mathrm{a} \in \mathrm{Jss}, \mathrm{b} \in \mathrm{Jss}, \mathrm{a} \geq 3, \mathrm{~b} \geq 3$

$(a+b) \geq 6,(a+b) / 2 \geq 3$,

$(\mathrm{a}+\mathrm{b}) / 2 \in \mathrm{N}^{+} \geq 3$

(1) $\mathrm{y}=\mathrm{x}$ In $[0,+\infty)$ in grade, neralized integral of Sa for infinite range of integration, $\sqrt{ } A_{1}=(a+b) / 2, \sqrt{ } A_{1} \in\left(N^{+} \geqslant 2\right.$, xist by $A$ for the elements of infinite sets, this infinite set of $A_{1}$ elements (2) (a quarter fa si e area $\mathrm{A}_{1}$ generalized integral value of the square root of 2 times) and $2(\mathrm{~N}+3)$ or mo the elen same.

(2) $2 \sqrt{A_{1}} \in 2\left(N^{+} \leq 3\right), W^{w}$ thin I quà arant, $\left.A 1,2\right)$ and $2(N+3)$ or more elements exist within the infinite sets one one corres onding and equal relationship.

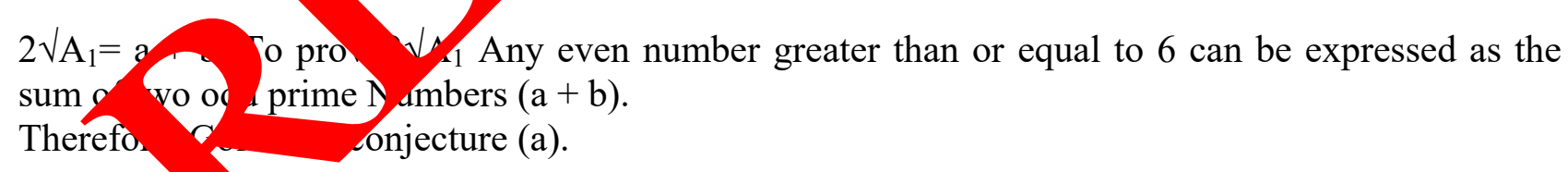

2.6 in the sai way to prove: Within the I quadrant, any odd integer greater than or equal to 9, can be expressed as the sum of three odd prime number $(a+b+c)$

$1,(a+b+c+1)$ is derived equations for a side length of square area.

Take $\mathrm{A}$ look at as A special curved trapezoid, $\mathrm{y}=\mathrm{x}$ in the interval $[0,+1)(\mathrm{A}+\mathrm{b}+\mathrm{c}]$ on integrable, The known conditions:

$\mathrm{A}=2 \mathrm{~S} 1 ; \mathrm{a} \geq 3, \mathrm{~b} \geq 3, \mathrm{c} \geq 3, \mathrm{a} \in \mathrm{Jss}, \mathrm{b} \in \mathrm{Jss}, \mathrm{c} \in \mathrm{Jss},(\mathrm{a}+\mathrm{b}+\mathrm{c}) \geq 9,(\mathrm{a}+\mathrm{b}+\mathrm{c}+1) \geq 10 ;(\mathrm{a}+\mathrm{b}+\mathrm{c}+1) \in 2$ $\left(\mathrm{N}^{+} \geqq 5\right), \mathrm{y}=(\mathrm{a}+\mathrm{b}+\mathrm{c}+1) ; \mathrm{x}=(\mathrm{a}+\mathrm{b}+\mathrm{c}+1) ; \mathrm{y}=\mathrm{x} ; \mathrm{A}=\mathrm{S}_{1}+\mathrm{S}_{2}$

A With definite integral is expressed as:

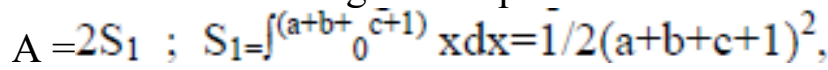

$\mathrm{A}=2 \int_{0}^{(\mathrm{a}+\mathrm{b}+\mathrm{c}+1)} \mathrm{xdx}=(\mathrm{a}+\mathrm{b}+\mathrm{c}+1)^{2}$ 
Proof: A in $[0,+\infty)$ as the limit of infinite generalized integral.

Proof:

Known:

$1, y=x$ is the elementary function, define the interval $[0,+$ up) for continuous function, meet the function continuous condition.

2. $a \geq 3, b \geq 3, c \geq 3, a \in J s s, b \in$ Jss, $c \in$ Jss, $(a+b+c) \geq 9,(a+b+c+1) \geq 10$, $(\mathrm{a}+\mathrm{b}+\mathrm{c}+1) \in 2\left(\mathrm{~N}^{+} \geqq 5\right), \mathrm{Y}=\mathrm{x}$ function $\mathrm{F}(\mathrm{x})=1 / 2(\mathrm{a}+\mathrm{b}+\mathrm{c}+1) 2$,

[function value $(\mathrm{a}+\mathrm{b}+\mathrm{c}+1) 2) / 2$ is of 50 or more constant. $(\operatorname{limC}=\mathrm{C})$;

By $y=(a+b+c+1), x=(a+b+c+1)$ and $y=x$ of a right triangle $S_{1}$ of the generalizantegral, The function $\mathrm{y}=\mathrm{x}$ in $[0,+\infty)$ on the generalized integral.

$\mathrm{A}=2 \mathrm{~S}_{1}$

$$
\int_{0}^{+\infty} \mathrm{xdx}=\lim \int_{0}^{\left(\mathrm{a}+\mathrm{b}+{ }_{0} \mathrm{c}+1\right)} \mathrm{xdx}=\lim \mathrm{x}^{2} / 2=\lim 1 / 2(\mathrm{a}+\mathrm{b}+\mathrm{c}+1)^{2}=1 / 2(\mathrm{a}+\mathrm{a}+1)^{2}
$$

$\mathrm{S} 1=\quad(\mathrm{a}+\mathrm{b}+\mathrm{c}+1) \rightarrow+\infty \mathrm{x} \rightarrow+\infty \quad(\mathrm{a}+\mathrm{b}+\mathrm{c}+1) \rightarrow+\infty$

$$
2 \int_{0}^{+\infty} x d x=\lim \int^{(a+b+c+1)} 2 x d x=\lim \left(x^{2} / 2\right) * 2=\lim (a+b+c
$$

$\mathrm{A}=$

$=(a+b+c+1)^{2}$

$$
(\mathrm{a}+\mathrm{b}+\mathrm{c}+1) \rightarrow+\infty \quad \mathrm{x} \rightarrow+\infty \quad(\mathrm{a}+\mathrm{b}+\mathrm{c}+1)
$$

Conclusion 2:

Within the I quadrant, the sum of any three odd prime umber, plus $1,(a+b+c+1)$ for the side length of square Area of A generalized integral value is finite.

2.7 Within I quadrant to $(a+b+c+\square 2$ is tilc midpoint of a side length of the coordinatevalues.

Will A is divided into four

A small square, as shown in figure

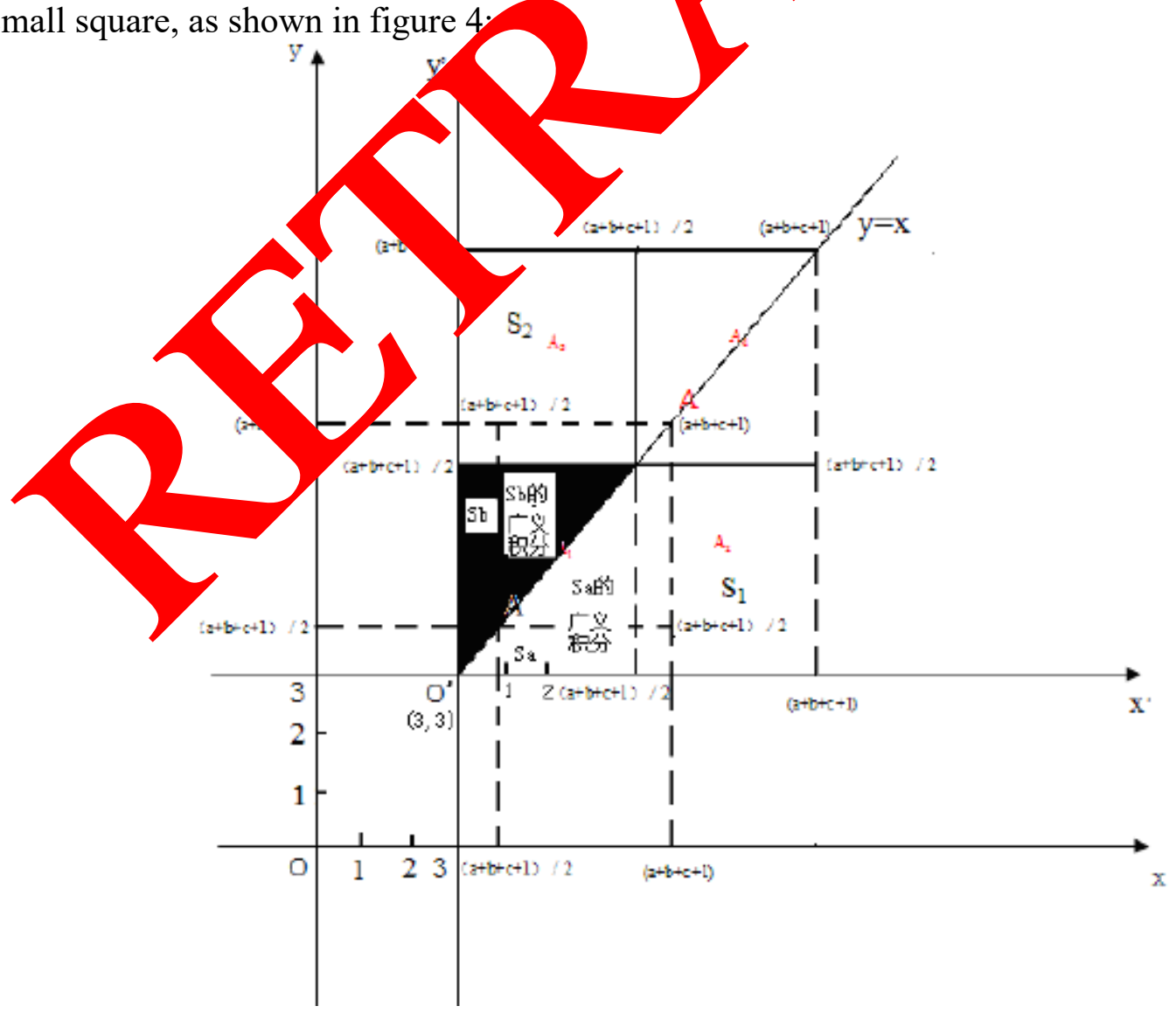

Figure - 4 
Because of the $2 \sqrt{ } \mathrm{A} 1-1=(\mathrm{a}+\mathrm{b}+\mathrm{c})$ Meet the known conditions: $\sqrt{\mathrm{A}_{1}}=(\mathrm{a}+\mathrm{b}+\mathrm{c}+1) / 2$, $\mathrm{a} \in \mathrm{Jss}, \mathrm{b} \in \mathrm{Jss}, \mathrm{c} \in \mathrm{Jss} \mathrm{a} \geq 3, \mathrm{~b} \geq 3, \mathrm{c} \geq 3$,

$(a+b+c) \geq 9,(a+b+c+1) / 2 \geq 5,(a+b+c+1) / 2 \in N^{+} \geq 5$

(1) $y=x \operatorname{In}[0,+\infty)$ is integral and the Sa for an improper integral, there by $\left.A_{1}-1\right)(2)$ for the element of infinite sets, this infinite set of elements (a quarter of a square area $A_{1}$ generalized integral value of the square root of $2 \times$ minus 1 )

Elements within a "Js 9 or higher" one to one correspondence and be equal.

(2) $\left(2 \sqrt{ } A_{1}-1\right) \in J_{S} \geq 9$ Within the I quadrant $\left(2 \sqrt{ } A_{1}-1\right)$ and $J_{s} \geq 9$ Infinite elements within a collection There is a one-to-one correspondence and equal relationship $2 \sqrt{ } A_{1}-1=(a+b+c)$ To prover $\left./ A_{1}-1\right)$ Is any odd number greater than or equal to 9 can be expressed as the sum of three odd $r$ ime $\mathrm{N}$. $(a+b+c)$. Therefore, Goldbach conjecture (b).

Conclusion Goldbach conjecture proposed since 1742, more mathemat cians pub d amost primes, exceptions to the rule set, such as three prime number theorem roof $y$ hods, als paper gives the create double rectangular coordinate system, using any two $\mathrm{r}$, the su of a prime number $(a+b) /(a+b+c)$ side to form a square with an area of a or a quar f a quare area A1 "square" diagonal integral method, derive the equations $2 \sqrt{A_{1}}=(\mathbf{a}+\mathbf{b}) ;\left(2 \sqrt{ } A_{1}-1\right)=(a+b+c) ;$

This proved that the innovation of the Goldbach conject re.

\section{Author's brief introduction:}

1. Ren Yongxue. (1965 -) male; Mongolian; to to the utilize modern stone oil company; Research direction: Proof of Goldbach conjectu e.
goldbach conjecture. yksryx@163.com 1 yksryx@163.com < mailto: proof of

2. Ren Yi (1990 -) female, Mongol an nà nality, angdong college of pharmacy students.

\section{REFERENCES:}

[1] Jiang Lu, Zhao Dip bai, er, Zhan Wan yu, li and zhang, shen revival, Chen Fumin China children's encyclope of scie technology (first edition), hangzhou: zhejiang education press, 1991, 145 Editori committee of o nomic mathematics.

[2] The central ia and $\mathrm{T}$ university. The economic applied mathematics (a) calculus (first edition) $P$ : the adio and Television University press, 1986. 299 - 300. 